MPI-PhT/96-23

UNIGRAZ-UTP-03-04-96

hep-lat/9604002

\title{
Lattice Regularization of the Chiral Schwinger Model
}

\author{
Christof Gattringer* \\ Max-Planck-Institut für Physik (Werner-Heisenberg-Institut) \\ Föhringer Ring 6, 80805 Munich, Germany \\ and \\ Institut für Theoretische Physik der Universität Graz \\ Universitätsplatz 5, 8010 Graz, Austria
}

\begin{abstract}
We analyze the chiral Schwinger model on an infinite lattice using the continuum definition of the fermion determinant and a linear interpolation of the lattice gauge fields. For non-compact and Wilson formulation of the gauge field action it is proven that the effective lattice model is Osterwalder-Schrader positive, which is a sufficient condition for the reconstruction of a physical Hilbert space from the model defined on a Euclidean lattice. For the non-compact model we furthermore establish the existence of critical points where the corresponding continuum theory can be reconstructed. We show that the continuum limit for the two-point functions of field strength and chiral densities can be controlled analytically. The article ends with some remarks on fermionic observables.
\end{abstract}

PACS number: 11.15.H

Keywords: lattice regularization, chiral fermions

* e-mail: chg@mppmu.mpg.de 


\section{Introduction}

It has been known since the first days of lattice field theory [1] that the regularization of chiral field theories on a lattice is a notoriously difficult problem (see [2] for a recent review). The celebrated Nielsen-Ninomya theorem [3] states that under reasonable assumptions (e.g locality of the lattice action) the number of left-handed fermions equals the number of right-handed fermions in a fermionic lattice theory. Thus it seems impossible to regularize chiral field theories as e.g. the electroweak sector of the standard model on the lattice.

In [4] 't Hooft renewed the interest in an old, alternative approach [5]- [14]. The idea is to put only the gauge field on the lattice, interpolate the gauge field to the interior of the lattice cells and couple the interpolated gauge field to continuum fermions. This hybrid approach 1 has many advantages. The number of degrees of freedom for the gauge fields remains finite (or countably infinite) and one can construct a measure for the gauge fields, which is (to the knowledge of the author) not possible directly in the continuum except for the gauge group U(1) (Gaussian measures). The Nielsen-Ninomya theorem is circumvented, since the continuum fermions do not have a local action from a lattice point of view. The hard part of the hybrid approach is to find a proper and explicit definition of the continuum fermion determinant in the interpolated background field. In [4] it is argued that for the U(1) gauge theory in 4 dimensions with vectorlike coupling to the fermions the continuum determinant in the interpolated background field can be given a meaning, and that all chiral symmetries are kept intact. This is an important result, but unfortunately the construction is not explicit, and it is not possible to explore details analytically. However it has to be remarked that similar, more explicit constructions using a finer grid for the fermions are being studied [12].

When proposing such a conceptionally new approach, one has to analyze its fundamental properties that allow to get back to a continuum theory in Minkowski space. In particular one is interested in proving Osterwalder-Schrader (OS) positivity 15 which allows the reconstruction of the physical Hilbert space and Hamiltonian from a theory with Euclidean metric. Furthermore one has to establish the existence of critical points with diverging correlation length, where the continuum limit can be performed. Analyzing such properties in four dimensional theories is not possible at the moment, in particular since explicit results for the fermion determinant in the interpolated background field are not available yet. However it is already desirable to see the hybrid approach work in simple models which can be controlled analytically. In 13] it was shown that for the hybrid approach to the vectorlike Schwinger model a critical point exists where the continuum limit can be performed. It was furthermore established that the chiral symmetries are kept intact and that no doubling occurs. A proof of OS positivity for the vectorlike model was given in [14].

The next logical step is to test the hybrid approach in a chiral model. A perfect candidate is the chiral Schwinger model first analyzed in [16] - [19]. The chiral Schwinger model in the continuum is under good analytic control, in particular the fermion determinant is known [16]. Results from the hybrid approach can be compared to their continuum counterparts explicitly. It has to be remarked that [7] attempted to set up the hybrid approach in this model long before the work of 't Hooft 㭵.

In this paper we apply the hybrid approach to the chiral Schwinger model. We use an interpolation scheme [5, 8, 13] which was already successfully implemented in the vectorlike

\footnotetext{
${ }^{1}$ The author partly ows the term "hybrid approach" to R. L. Stuller who christened his approach "Hybrid Quantization".
} 
model [13, 14]. The Jackiw-Rajaraman determinant [16] is used to construct the effective lattice gauge theory (Section 2). In Section 3 we prove that the resulting effective lattice gauge model is OS positive for both the non-compact and the Wilson formulation of the gauge field action. In Section 4 we establish the existence of critical points and perform the continuum limit for the two point function of the field strength. Section 5 is devoted to the discussion of fermionic observables. Section 6 contains concluding remarks.

\section{Interpolation and the effective lattice action}

The lattice under consideration is $\mathbb{Z}^{2}$, i.e. the lattice spacing is set to one. Functions defined in the continuum can be identified by their arguments $x, y \in \mathbb{R}^{2}$, while lattice quantities have arguments $n, m \in \mathbb{Z}^{2}$. We consider two types of actions for the $\mathrm{U}(1)$-gauge fields. The noncompact action is given by

$$
S_{n c}:=\frac{1}{4} \sum_{n \in \mathbb{Z}^{2}} F_{\mu \nu}(n) F_{\mu \nu}(n)=\frac{1}{2} \sum_{n \in \mathbb{Z}^{2}} F_{12}(n)^{2},
$$

with

$$
F_{\mu \nu}(n):=\left(A_{\nu}\left(n+\hat{e}_{\mu}\right)-A_{\nu}(n)-A_{\mu}\left(n+\hat{e}_{\nu}\right)+A_{\mu}(n)\right) .
$$

$A_{\mu}(n)$ may assume values in $(-\infty,+\infty)$ for all $n \in \mathbb{Z}^{2}$ and $\mu=1,2$. $\hat{e}_{\mu}$ is the unit vector in $\mu$-direction. We also consider the Wilson formulation of the gauge field action

$$
S_{w}:=\frac{1}{e^{2}} \sum_{n \in \mathbb{Z}^{2}}\left[1-\operatorname{Re}\left(U_{1}(n) U_{2}\left(n+\hat{e}_{1}\right) \overline{U_{1}\left(n+\hat{e}_{2}\right) U_{2}(n)}\right)\right]=\frac{1}{e^{2}} \sum_{n \in \mathbb{Z}^{2}}\left[1-\operatorname{Re} e^{i e F_{12}(n)}\right] .
$$

In the last step the gauge transporters were expressed as

$$
U_{\mu}(n):=\exp \left(i e A_{\mu}(n)\right) \text { with } e A_{\mu}(n) \in[0,2 \pi) .
$$

Although we use the same symbol, it has to be kept in mind that the $A_{\mu}(n)$ are restricted to the principal branch $[0,2 \pi / e)$ in the case of the Wilson action.

We interpolate the fields as follows [5, 8, 13]

$$
A_{1}^{i n t}(x):=A_{1}(n)\left[1-t_{2}\right]+A_{1}\left(n+\hat{e}_{2}\right) t_{2} \quad, \quad A_{2}^{\text {int }}(x):=A_{2}(n)\left[1-t_{1}\right]+A_{2}\left(n+\hat{e}_{1}\right) t_{1},
$$

for $x=n+t$ and $t_{1}, t_{2} \in(0,1]$. This type of interpolation was already used for the treatment of the vectorlike Schwinger model on the lattice [5, 13]. In [13] it was shown that the interpolation (2.5) respects the condition of gauge equivariance (i.e. transforming a lattice gauge transformation to a continuum gauge transformation for the interpolated fields).

For later use we quote the (continuum) Fourier transform of the interpolated fields

$$
\begin{aligned}
& \widetilde{A}_{1}^{i n t}(p)=\int_{-\infty}^{\infty} d^{2} x A_{1}(x) e^{-i p x}=: \widehat{A}_{1}(p) \frac{1-e^{-i p_{1}}}{i p_{1}} \frac{2-2 \cos \left(p_{2}\right)}{p_{2}^{2}}, \\
& \widetilde{A}_{2}^{\text {int }}(p)=\int_{-\infty}^{\infty} d^{2} x A_{2}(x) e^{-i p x}=: \widehat{A}_{2}(p) \frac{1-e^{-i p_{2}}}{i p_{2}} \frac{2-2 \cos \left(p_{1}\right)}{p_{1}^{2}}
\end{aligned}
$$


where we introduced the lattice Fourier transform

$$
\widehat{A}_{\mu}(p):=\sum_{n \in \mathbb{Z}^{2}} e^{-i p n} A_{\mu}(n) .
$$

The continuum Fourier transform $\widetilde{A}_{\mu}^{\text {int }}$ of the interpolated lattice fields comes out as the Fourier transform on the lattice $\widehat{A}_{\mu}$ multiplied by some function which depends on the details of the interpolation and explicitly shows the ultraviolet regulator (inverse powers of $p_{\mu}$ ) which is introduced by the lattice.

The first step in the hybrid approach is to give a meaning to the determinant in the continuum. The Euclidean continuum action for the fermions is given by

$$
S_{F}:=\int_{-\infty}^{\infty} d^{2} x \bar{\chi}(x)\left[\partial_{1}-i \partial_{2}-i e\left(A_{1}(x)-i A_{2}(x)\right)\right] \chi(x)
$$

where $\bar{\chi}, \chi$ are left handed Weyl fermions. It has to be remarked, that often (see e.g. [16])) the chiral Schwinger model is defined using Dirac spinors, where only one chiral component couples to the gauge field. Of course the results are the same, since the other component decouples. The well known result for the regularized fermion determinant is given by [16] (see e.g. [20] for the translation to the Euclidean version quoted here)

$$
\operatorname{det}_{r e g}\left[\partial_{1}-i \partial_{2}-i e\left(A_{1}-i A_{2}\right)\right]=\exp \left(-\frac{1}{2} g^{2} \int_{-\infty}^{\infty} \frac{d^{2} p}{(2 \pi)^{2}} \frac{1}{p^{2}} \widetilde{A}_{\mu}(-p) \widetilde{M}_{\mu \nu}(p) \widetilde{A}_{\nu}(p)\right)=: \exp (-W[A])
$$

where

$$
\widetilde{M}_{\mu \nu}(p)=(a+1) \delta_{\mu \nu} p^{2}-2 p_{\mu} p_{\nu}+i\left[p_{\mu} \varepsilon_{\rho \nu} p_{\rho}+p_{\rho} \varepsilon_{\rho \mu} p_{\nu}\right] .
$$

$g^{2}$ is defined as $g^{2}:=e^{2} / 4 \pi$. The Jackiw-Rajaraman parameter $a$ parametrizes an ambiguity in the regularization of a short distance singularity. An explicit computation of the determinant directly in Euclidean space, following [21] can be found in [7]. There the regularization is performed such that $a=1$. The full freedom in the regularization of the short distance singularity is e.g. analyzed in 22].

The effective action $W[A]$ defined through $(2.9),(2.10)$ is complex and cannot be made gauge invariant by adjusting $a$. It has to be remarked, that for $a=1$ the kernel of the quadratic form in the gauge fields $W[A]$ cannot be inverted, and the propagator for the gauge fields does not exist then. For $a<1$, the model contains ghosts [16].

The next step in the hybrid approach for formulating the chiral model on the lattice is to plug the interpolated gauge fields (2.6) into the expression (2.9), (2.10) for the continuum determinant giving rise to

$$
W^{l a t}[A]:=W\left[A^{i n t}\right]=\frac{1}{2} g^{2} \int_{-\infty}^{\infty} \frac{d^{2} p}{(2 \pi)^{2}} \frac{2-2 \cos \left(p_{1}\right)}{p_{1}^{2}} \frac{2-2 \cos \left(p_{2}\right)}{p_{2}^{2}} \frac{1}{p^{2}} \widehat{A}_{\mu}(-p) \widehat{M}_{\mu \nu}(p) \widehat{A}_{\nu}(p),
$$

where the kernel $\widehat{M}_{\mu \nu}$ for the lattice fields $\widehat{A}_{\mu}$ is given by

$$
\begin{aligned}
& \widehat{M}_{11}(p)=\left[(a+1) p^{2}-2 p_{1}^{2}-i 2 p_{1} p_{2}\right] \frac{2-2 \cos \left(p_{2}\right)}{p_{2}^{2}} \\
& \widehat{M}_{12}(p)=\left[-2 p_{1} p_{2}+i\left(p_{1}^{2}-p_{2}^{2}\right)\right] \frac{\left(e^{i p_{1}}-1\right)\left(e^{-i p_{2}}-1\right)}{p_{1} p_{2}}
\end{aligned}
$$




$$
\begin{aligned}
& \widehat{M}_{21}(p)=\left[-2 p_{1} p_{2}+i\left(p_{1}^{2}-p_{2}^{2}\right)\right] \frac{\left(e^{-i p_{1}}-1\right)\left(e^{i p_{2}}-1\right)}{p_{1} p_{2}} \\
& \widehat{M}_{22}(p)=\left[(a+1) p^{2}-2 p_{2}^{2}+i 2 p_{1} p_{2}\right] \frac{2-2 \cos \left(p_{1}\right)}{p_{1}^{2}}
\end{aligned}
$$

For later use we quote the following symmetry properties of $\widehat{M}_{\mu \nu}$

$$
\begin{gathered}
\widehat{M}_{\mu \mu}\left(-p_{1}, p_{2}\right)=\widehat{M}_{\mu \mu}\left(p_{1},-p_{2}\right)=\widehat{\widehat{M}}_{\mu \mu}\left(p_{1}, p_{2}\right) \quad, \quad \mu=1,2, \\
\widehat{M}_{12}\left(-p_{1},-p_{2}\right)=\widehat{M}_{21}\left(p_{1}, p_{2}\right), \\
-e^{-i p_{2}} \overline{\widehat{M}_{21}\left(p_{1},-p_{2}\right)}=\widehat{M}_{12}\left(p_{1}, p_{2}\right) \quad, \quad-e^{i p_{2}} \overline{\widehat{M}_{12}\left(p_{1},-p_{2}\right)}=\widehat{M}_{21}\left(p_{1}, p_{2}\right) .
\end{gathered}
$$

Adding the contribution $W^{\text {int }}$ from the fermion determinant to the lattice action for the gauge fields $S_{n c}, S_{w}$ respectively gives the effective lattice action for the chiral Schwinger model in non-compact and Wilson formulation

$$
S_{n c}^{e f f}:=S_{n c}+W^{l a t} \quad, \quad S_{w}^{e f f}:=S_{w}+W^{l a t} .
$$

\section{Proof of OS positivity}

Osterwalder and Schrader [15] developed a mathematical procedure that allows the reconstruction of the Hamiltonian and the physical Hilbert space from a continuum field theory defined in Euclidean space. The main condition is Osterwalder-Schrader (OS) positivity (see below). For the Wilson formulation of lattice gauge theory, OS positivity was shown to hold by Osterwalder and Seiler [23]. For the hybrid approach a new proof has to be given.

For this purpose, we decompose the field algebra into three $\operatorname{disjoint}$ sets $\mathcal{A}^{+}, \mathcal{A}^{0}$ and $\mathcal{A}^{-}$, defined as

$$
\begin{aligned}
\mathcal{A}^{+} & :=\left\{A_{1}\left(n_{1}, n_{2}\right), A_{2}\left(n_{1}, m_{2}\right) \mid n_{1} \in \mathbb{Z}, n_{2}>1, m_{2}>1\right\}, \\
\mathcal{A}^{0} & :=\left\{A_{1}\left(n_{1}, 1\right), A_{1}\left(n_{1}, 0\right), A_{2}\left(n_{1}, 1\right), A_{2}\left(n_{1}, 0\right), A_{2}\left(n_{1},-1\right) \mid n_{1} \in \mathbb{Z}\right\}, \\
\mathcal{A}^{-} & :=\left\{A_{1}\left(n_{1}, n_{2}\right), A_{2}\left(n_{1}, m_{2}\right) \mid n_{1} \in \mathbb{Z}, n_{2}<0, m_{2}<-1\right\} .
\end{aligned}
$$

We furthermore define the antilinear time reflection operator $\Theta$ acting on the gauge fields as follows

$$
\Theta A_{1}\left(n_{1}, n_{2}\right):=A_{1}\left(n_{1},-n_{2}+1\right) \quad, \quad \Theta A_{2}\left(n_{1}, n_{2}\right):=-A_{2}\left(n_{1},-n_{2}\right) .
$$

It is easy to check that $\Theta$ maps $\mathcal{A}^{+}$onto $\mathcal{A}^{-}$and $\mathcal{A}^{0}$ onto itself. The condition of OS positivity is now defined as

$$
\left\langle P\left[\mathcal{A}^{+}\right] \Theta P\left[\mathcal{A}^{+}\right]\right\rangle \geq 0 \quad, \quad \forall P\left[\mathcal{A}^{+}\right]
$$

where $P\left[\mathcal{A}^{+}\right]$denotes an arbitrary function depending only on the field variables in $\mathcal{A}^{+}$. Following [23, 24] we show that both effective actions (2.16) can be decomposed as follows

$$
-S^{e f f}=-S^{+}\left[\mathcal{A}^{+}, \mathcal{A}^{0}\right]-\Theta S^{+}\left[\mathcal{A}^{+}, \mathcal{A}^{0}\right]+\int d \mu[\lambda] C_{\lambda}\left[\mathcal{A}^{+}\right] \Theta C_{\lambda}\left[\mathcal{A}^{+}\right],
$$

where $d \mu[\lambda]$ denotes some positive measure, and $S^{+}\left[\mathcal{A}^{+}, \mathcal{A}^{0}\right]$ depends only on the field variables in $\mathcal{A}^{+} \cup \mathcal{A}^{0}$ and $C_{\lambda}\left[\mathcal{A}^{+}\right]$only on the variables in $\mathcal{A}^{+}$. The decomposition (3.4) is a sufficient 
condition for (3.3) to hold, since by expanding the exponential of the last term in (3.4) one obtains (use the antilinearity of $\Theta$ )

$$
\left\langle P\left[\mathcal{A}^{+}\right] \Theta P\left[\mathcal{A}^{+}\right]\right\rangle=\frac{1}{Z} \int D\left[\mathcal{A}^{0}\right] \sum_{j=0}^{\infty} \frac{1}{j !} \int \prod_{l=1}^{j} d \mu\left[\lambda_{l}\right]\left|\int D\left[\mathcal{A}^{+}\right] e^{-S^{+}\left[\mathcal{A}^{+}, \mathcal{A}^{0}\right]} P\left[\mathcal{A}^{+}\right] \prod_{i=1}^{j} C_{\lambda_{i}}\left[\mathcal{A}^{+}\right]\right|^{2} \geq 0 .
$$

For the two expressions $S_{n c}, S_{w}$ of the gauge field part of the action, it is well known, that a decompositions of the type (3.4) exists [23, 14]. Using $\Theta F_{12}\left(n_{1}, n_{2}\right)=-F_{12}\left(n_{1},-n_{2}\right)$, which follows from (3.2), one finds

$$
-S_{n c}=-S_{n c}^{+}-\Theta S_{n c}^{+} \quad, \quad-S_{w}=-S_{w}^{+}-\Theta S_{w}^{+},
$$

where

$$
S_{n c}^{+}:=\frac{1}{2} \sum_{n_{1} \in \mathbb{Z}, n_{2}>0} F_{12}\left(n_{1}, n_{2}\right)^{2}+\frac{1}{4} \sum_{n_{1} \in \mathbb{Z}} F_{12}\left(n_{1}, 0\right)^{2}
$$

and

$$
S_{w}^{+}:=\frac{1}{e^{2}} \sum_{n_{1} \in \mathbb{Z}, n_{2}>0}\left[1-\operatorname{Re} e^{i e F_{12}\left(n_{1}, n_{2}\right)}\right]+\frac{1}{2 e^{2}} \sum_{n_{1} \in \mathbb{Z}}\left[1-\operatorname{Re} e^{i e F_{12}\left(n_{1}, 0\right)}\right] .
$$

Thus there is only the contribution $W^{\text {lat }}[A]$ from the fermion determinant left to analyze. Using (3.2), and the antilinearity of $\Theta$, we decompose the Fourier transform (2.7) of the gauge field components as

$$
\begin{aligned}
& \widehat{A}_{1}\left(p_{1}, p_{2}\right)=\widehat{A}_{1}^{+}\left(p_{1}, p_{2}\right)+\widehat{A}_{1}^{0}\left(p_{1}, p_{2}\right)+e^{-i p_{2}} \Theta \widehat{A}_{1}^{+}\left(-p_{1}, p_{2}\right), \\
& \widehat{A}_{2}\left(p_{1}, p_{2}\right)=\widehat{A}_{2}^{+}\left(p_{1}, p_{2}\right)+\widehat{A}_{2}^{0}\left(p_{1}, p_{2}\right)-\Theta \widehat{A}_{2}^{+}\left(-p_{1}, p_{2}\right),
\end{aligned}
$$

where we defined

$$
\widehat{A}_{\mu}^{+}(p):=\sum_{n_{1} \in \mathbb{Z}, n_{2}>1} e^{-i p n} A_{\mu}(n), \quad \mu=1,2,
$$

and

$$
\begin{aligned}
& \widehat{A}_{1}^{0}\left(p_{1}, p_{2}\right):=\sum_{n_{1} \in \mathbb{Z}} e^{-i p_{1} n_{1}}\left[e^{-i p_{2}} A_{1}\left(n_{1}, 1\right)+A_{1}\left(n_{1}, 0\right)\right] \\
& \widehat{A}_{2}^{0}\left(p_{1}, p_{2}\right):=\sum_{n_{1} \in \mathbb{Z}} e^{-i p_{1} n_{1}}\left[e^{-i p_{2}} A_{2}\left(n_{1}, 1\right)+A_{1}\left(n_{1}, 0\right)+e^{i p_{2}} A_{2}\left(n_{1},-1\right)\right] .
\end{aligned}
$$

For later use we note the reflection properties of the $\widehat{A}_{\mu}^{0}$ which follow from (3.2) and the antilinearity of $\Theta$

$$
\Theta \widehat{A}_{1}^{0}\left(p_{1}, p_{2}\right)=e^{i p_{2}} \widehat{A}_{1}^{0}\left(-p_{1}, p_{2}\right) \quad, \quad \Theta \widehat{A}_{2}^{0}\left(p_{1}, p_{2}\right)=-\widehat{A}_{2}^{0}\left(-p_{1}, p_{2}\right) .
$$

Inserting (3.9) and (3.10) into (2.11) gives, after writing explicitly the sum over $\mu$ and $\nu$, altogether 36 terms. Some of them are equal, some are the image of others under the reflection $\Theta$. Using (3.14), the properties (2.13)-(2.15) of $\widehat{M}_{\mu \nu}$, the antilinearity of $\Theta$ and coordinate transformations of the type $p_{\mu} \rightarrow-p_{\mu}$ in the integrals over the momentum space, one obtains after a lengthy but straightforward computation

$$
-W^{l a t}[A]=-W[A]^{+}-\Theta W[A]^{+}+W^{m i x}[A],
$$


with

$$
\begin{gathered}
W[A]^{+}:=\frac{g^{2}}{4} \int_{-\infty}^{\infty} \frac{d^{2} p}{(2 \pi)^{2}} \frac{1}{p^{2}} \frac{2-2 \cos \left(p_{1}\right)}{p_{1}^{2}} \frac{2-2 \cos \left(p_{2}\right)}{p_{2}^{2}} \\
\times\left[2 \widehat{A}_{\mu}^{+}(-p) \widehat{M}_{\mu \nu}(p) \widehat{A}_{\nu}^{+}(p)+4 \widehat{A}_{\mu}^{0}(-p) \widehat{M}_{\mu \nu}(p) \widehat{A}_{\nu}^{+}(p)+\widehat{A}_{\mu}^{0}(-p) \widehat{M}_{\mu \nu}(p) \widehat{A}_{\nu}^{0}(p)\right] .
\end{gathered}
$$

Thus (compare (3.4) and (3.15)) there is only left to show, that $W^{\text {mix }}[A]$ has the form of the last term in (3.4). $W^{\text {mix }}[A]$ is given by

$$
W^{\text {mix }}[A]:=g^{2} \int_{-\infty}^{\infty} \frac{d^{2} p}{(2 \pi)^{2}} \frac{1}{p^{2}} \frac{2-2 \cos \left(p_{1}\right)}{p_{1}^{2}} \frac{2-2 \cos \left(p_{2}\right)}{p_{2}^{2}}\left[\Theta \widehat{A}_{\mu}^{+}\left(p_{1},-p_{2}\right)\right] \widehat{R}_{\mu \nu}\left(p_{1}, p_{2}\right) \widehat{A}_{\nu}^{+}\left(p_{1}, p_{2}\right)
$$

where

$$
\begin{aligned}
\widehat{R}_{11}(p) & :=\left[-(a+1) p^{2}+2\left(p_{1}^{2}+i p_{1} p_{2}\right)\right] \frac{2-2 \cos \left(p_{2}\right)}{p_{2}^{2}} e^{i p_{2}} \\
\widehat{R}_{12}(p) & :=\left[2 p_{1} p_{2}-i\left(p_{1}^{2}-p_{2}^{2}\right)\right] \frac{\left(e^{i p_{1}}-1\right)\left(e^{-i p_{2}}-1\right)}{p_{1} p_{2}} e^{i p_{2}} \\
\widehat{R}_{21}(p) & :=\left[-2 p_{1} p_{2}+i\left(p_{1}^{2}-p_{2}^{2}\right)\right] \frac{\left(e^{-i p_{1}}-1\right)\left(e^{i p_{2}}-1\right)}{p_{1} p_{2}} \\
\widehat{R}_{22}(p) & :=\left[(a+1) p^{2}-2\left(p_{2}^{2}-i p_{1} p_{2}\right)\right] \frac{2-2 \cos \left(p_{1}\right)}{p_{1}^{2}} .
\end{aligned}
$$

Inserting (3.11) into (3.17) one obtains (use (3.2), and the antilinearity of $\Theta$ )

$$
W^{m i x}[A]=\sum_{n_{1}, m_{1} \in \mathbb{Z}} \sum_{n_{2}, m_{2}>1}\left[\Theta A_{\mu}\left(m_{1}, m_{2}\right)\right] A_{\nu}\left(n_{1}, n_{2}\right) P_{\mu \nu}\left(m_{1}-n_{1}, m_{2}+n_{2}\right),
$$

where

$$
P_{\mu \nu}\left(k_{1}, k_{2}\right):=g^{2} \int_{-\infty}^{\infty} \frac{d p_{1}}{(2 \pi)^{2}} \frac{2-2 \cos \left(p_{1}\right)}{p_{1}^{2}} e^{i p_{1} k_{1}} \int_{-\infty}^{\infty} d p_{2} \frac{2-2 \cos \left(p_{2}\right)}{p_{2}^{2}} e^{-i p_{2} k_{2}} \frac{1}{p_{1}^{2}+p_{2}^{2}} \widehat{R}_{\mu \nu}\left(p_{1}, p_{2}\right) \text {. }
$$

The $p_{2}$ integration can be carried out using the residue theorem. We close the contour in the lower complex $p_{2}$-half plane. The factor $e^{-i p_{2} k_{2}}$ dominates the exponentials from the cosine and the exponents in $\widehat{R}_{\mu \nu}\left(p_{1}, p_{2}\right)$, since $k_{2}=m_{2}+n_{2} \geq 4$ as can be seen from the sum in (3.19). The only pole in the lower $p_{2}$-half plane is at $p_{2}=-i\left|p_{1}\right|$ and one obtains

$$
P_{\mu \nu}\left(k_{1}, k_{2}\right):=g^{2} \int_{-\infty}^{\infty} \frac{d p_{1}}{4 \pi} \frac{2-2 \cos \left(p_{1}\right)}{p_{1}^{2}} \frac{2 \cosh \left(p_{1}\right)-2}{p_{1}^{2}} e^{i p_{1} k_{1}} e^{-\left|p_{1}\right| k_{2}} \frac{1}{\left|p_{1}\right|} \widehat{R}_{\mu \nu}\left(p_{1},-i\left|p_{1}\right|\right) .
$$

Evaluating $\widehat{R}_{\mu \nu}\left(p_{1},-i\left|p_{1}\right|\right)$ gives the matrix

$$
\widehat{R}\left(p_{1},-i\left|p_{1}\right|\right)=4 \theta\left(p_{1}\right)\left[\begin{array}{cc}
\left(e^{p_{1}}-1\right)^{2} & -\left(e^{i p_{1}}-1\right)\left(e^{p_{1}}-1\right) \\
-\left(e^{-i p_{1}}-1\right)\left(e^{p_{1}}-1\right) & \left(e^{i p_{1}}-1\right)\left(e^{-i p_{1}}-1\right)
\end{array}\right],
$$

where $\theta$ denotes the Heavyside step-function. The resulting matrix is hermitean and thus can be diagonalized by some unitary matrix $U$. One finds

$$
\widehat{R}\left(p_{1},-i\left|p_{1}\right|\right)=4 \theta\left(p_{1}\right) U \operatorname{diag}\left(\left(e^{p_{1}}-1\right)^{2}+2-2 \cos \left(p_{1}\right), 0\right) U^{\dagger},
$$


where

$$
U:=\left[\left(e^{p_{1}}-1\right)^{2}+2-2 \cos \left(p_{1}\right)\right]^{-\frac{1}{2}}\left[\begin{array}{cc}
e^{p_{1}}-1 & e^{i p_{1}}-1 \\
1-e^{-i p_{1}} & e^{p_{1}}-1
\end{array}\right] .
$$

As can be seen from the explicit diagonalization (3.23), the matrix $\widehat{R}\left(p_{1},-i\left|p_{1}\right|\right)$ has a vanishing and a positive real eigenvalue. The semidefiniteness of this matrix allows to construct a positive measure $d \mu[\lambda]$ as needed for the last term in (3.4). Putting things together

$$
W^{\text {mix }}[A]=g^{2} \int_{0}^{\infty} \frac{d p_{1}}{\pi} \frac{2-2 \cos \left(p_{1}\right)}{p_{1}^{2}} \frac{2 \cosh \left(p_{1}\right)-2}{p_{1}^{2}}\left[\Theta f\left(p_{1}\right)\right] f\left(p_{1}\right),
$$

where we defined

$$
f\left(p_{1}\right)=\sum_{n_{1} \in \mathbb{Z}} \sum_{n_{2}>1} e^{-i p_{1} n_{1}} e^{-p_{1} n_{2}}\left[\frac{e^{p_{1}}-1}{p_{1}} A_{1}\left(n_{1}, n_{2}\right)-\frac{e^{i p_{1}}-1}{p_{1}} A_{2}\left(n_{1}, n_{2}\right)\right] .
$$

Together with (3.6), Equation (3.25) establishes the decomposition of $-W^{\text {lat }}[A]$ into the form (3.4) and thus OS positivity (3.3). OS positivity is the starting point of the Osterwalder-Schrader reconstruction [15] which leads to Hamiltonian and physical Hilbert space.

It is remarkable that the proof given does not restrict the values of the Jackiw-Rajaraman parameter $a$. This surprising result might be understood, by the fact that $a$ enters the continuum action (2.9), (2.10) for the gauge field only via a contact term, which is irrelevant for the Osterwalder-Schrader reconstruction in the continuum.

\section{Critical points and continuum limit}

In the case of the vectorlike Schwinger model, it turned out, that for the analysis of critical points it is most convenient to study the two point function of the field strength [13, 14]. Here we follow this strategy and compute

$$
E(n):=\left\langle F_{12}(n) F_{12}(0)\right\rangle
$$

For notational convenience we introduce new fields defined as

$$
\varphi_{1}(n):=A_{2}\left(n+\hat{e}_{1}\right)-A_{2}(n), \varphi_{2}(n):=A_{1}\left(n+\hat{e}_{2}\right)-A_{1}(n),
$$

which implies (compare (2.2))

$$
F_{12}(n)=\varphi_{1}(n)-\varphi_{2}(n)
$$

In momentum space one finds

$$
\widehat{\varphi}_{1}(p)=\left(e^{i p_{1}}-1\right) \widehat{A}_{2}(p) \quad, \quad \widehat{\varphi}_{2}(p)=\left(e^{i p_{2}}-1\right) \widehat{A}_{1}(p) .
$$

From now on we restrict ourself to the non-compact formulation (2.1) of the gauge field action. Rewriting it first to momentum space and then in terms of $\varphi_{\mu}$ one obtains for $S_{n c}$

$$
S_{n c}=\frac{1}{2} \int_{-\pi}^{\pi} \frac{d^{2} q}{(2 \pi)^{2}} \widehat{F}_{12}(-q) \widehat{F}_{12}(q)=\frac{1}{2} \int_{-\pi}^{\pi} \frac{d^{2} q}{(2 \pi)^{2}} \widehat{\varphi}_{\mu}(-q) P_{\mu \nu} \widehat{\varphi}_{\nu}(q),
$$


where $P_{\mu \nu}$ is defined as $P_{\mu \nu}:=2 \delta_{\mu \nu}-1$. Inserting (4.4) in (2.11) and adding the result to (4.5), one obtains the effective action $S_{n c}^{e f f}$ (see (3.6)) in terms of $\varphi_{\mu}$

$$
S_{n c}^{e f f}=\frac{1}{2} \int_{-\pi}^{\pi} \frac{d^{2} q}{(2 \pi)^{2}} \widehat{\varphi}_{\mu}(-q) \widehat{C}_{\mu \nu}^{-1}(q) \widehat{\varphi}_{\nu}(q),
$$

where

$$
\begin{aligned}
\widehat{C}_{11}^{-1}(q) & :=1+g^{2}\left(2-2 \cos \left(q_{1}\right)\right)\left(2-2 \cos \left(q_{2}\right)\right) \sum_{k \in \mathbb{Z}^{2}} \frac{1}{r_{1}^{4} r_{2}^{4} r^{2}}\left[(a+1) r_{2}^{2} r^{2}-2 r_{2}^{4}+i 2 r_{1} r_{2}^{3}\right], \\
\widehat{C}_{12}^{-1}(q) & :=-1+g^{2}\left(2-2 \cos \left(q_{1}\right)\right)\left(2-2 \cos \left(q_{2}\right)\right) \sum_{k \in \mathbb{Z}^{2}} \frac{1}{r_{1}^{4} r_{2}^{4} r^{2}}\left[-2 r_{1}^{2} r_{2}^{2}+i\left(r_{1}^{3} r_{2}-r_{1} r_{2}^{3}\right)\right], \\
\widehat{C}_{21}^{-1}(q) & :=\widehat{C}_{12}^{-1}(q), \\
\widehat{C}_{22}^{-1}(q) & :=1+g^{2}\left(2-2 \cos \left(q_{1}\right)\right)\left(2-2 \cos \left(q_{2}\right)\right) \sum_{k \in \mathbb{Z}^{2}} \frac{1}{r_{1}^{4} r_{2}^{4} r^{2}}\left[(a+1) r_{1}^{2} r^{2}-2 r_{1}^{4}-i 2 r_{1}^{3} r_{2}\right] .
\end{aligned}
$$

We introduced the abbreviation

$$
r_{\mu}:=q_{\mu}+2 \pi k_{\mu},
$$

where $k$ runs over $\mathbb{Z}^{2}$, i.e. over all Brillouin zones. This sum over all Brillouin zones comes from rewriting the momentum integral over $\mathbb{R}^{2}$ which shows up in (2.11) as a sum of integrals over the first Brillouin zone only (note that $\widehat{A}_{\mu}(q)$, and thus also $\widehat{\varphi}_{\mu}(q)$ are periodic with respect to shifting the argument to another Brillouin zone).

We quantize the model by performing the path integral over the fields $\varphi_{\mu}$. This gives rise to a Gaussian integral with covariance $C$ (see below). As in the continuum model (compare e.g. [16]), no gauge fixing term has to be added, since the non-gauge-invariant action (2.11) already fixes the gauge. In order to compute the covariance, we have to analyze for which values of $a$ $\widehat{C}^{-1}(q)$ can be inverted as a matrix. Define

$$
D(q):=\operatorname{det} \widehat{C}^{-1}(q)=: g^{2}(a-1) D_{1}(q)+g^{4} a^{2} D_{2}(q) .
$$

The terms $D_{j}(q), j=1,2$ are easy to compute but rather lengthy and we quote them in the Appendix. There it is also shown (Lemma 1) that at least for $a \geq 2$ the real part of $D(q)$ is strictly positive for all values of $q$ and for $g>0$. This establishes that $\widehat{C}^{-1}(q)$ can be inverted as a matrix. In the Appendix it is shown furthermore, that this result implies the existence of the propagator also for the original fields $A_{\mu}$ for $a \geq 2$. It has to be noticed that for the model in the continuum the determinant is real and positive for $a>1$. We were not able to extend the proof given in the Appendix to values $a>1$. However in the discussion of the continuum limit (see below), it will be shown that this difference in the range of $a$ is irrelevant.

The propagator $\widehat{C}(q)$ is given by

$$
\widehat{C}(q)=\frac{1}{D(q)}\left[\begin{array}{cc}
\widehat{C}_{22}^{-1}(q) & -\widehat{C}_{12}^{-1}(q) \\
-\widehat{C}_{12}^{-1}(q) & \widehat{C}_{11}^{-1}(q)
\end{array}\right] .
$$

Using (4.3) one finds

$$
E(n)=\left\langle\left[\varphi_{1}(n)-\varphi_{2}(n)\right]\left[\varphi_{1}(0)-\varphi_{2}(0)\right]\right\rangle=\int_{-\pi}^{\pi} \frac{d^{2} q e^{i q n}}{(2 \pi)^{2}}\left[\widehat{C}_{11}(q)+\widehat{C}_{22}(q)-2 \widehat{C}_{12}(q)\right]
$$




$$
\begin{gathered}
=\int_{-\pi}^{\pi} \frac{d^{2} q e^{i q n}}{(2 \pi)^{2}} \frac{g^{2}(a-1)}{D(q)} \sum_{k \in \mathbb{Z}^{2}} \frac{2-2 \cos \left(q_{1}\right)}{r_{1}^{2}} \frac{2-2 \cos \left(q_{2}\right)}{r_{2}^{2}} \frac{r^{2}}{r_{1}^{2} r_{2}^{2}} \\
=\int_{-\pi}^{\pi} \frac{d^{2} q e^{i q n}}{(2 \pi)^{2}} \frac{g^{2}(a-1) D_{1}(q)}{g^{2}(a-1) D_{1}(q)+g^{4} a^{2} D_{2}(q)} .
\end{gathered}
$$

In the last step we used the definition of $D_{1}(q)$ (Equation(A.2)) and inserted (4.9) for $D(q)$. Rewriting the integrand one obtains

$$
E(n)=\int_{-\pi}^{\pi} \frac{d^{2} q e^{i q n}}{(2 \pi)^{2}}\left[1-m^{2} \frac{1}{\sigma(q)+m^{2}}\right]=\delta_{n_{1}, 0} \delta_{n_{2}, 0}-m^{2} \int_{-\pi}^{\pi} \frac{d^{2} q}{(2 \pi)^{2}} \frac{e^{i q n}}{\sigma(q)+m^{2}},
$$

where we defined

$$
m^{2}:=g^{2} \frac{a^{2}}{a-1}
$$

and

$$
\sigma(q):=\frac{D_{1}(q)}{D_{2}(q)}
$$

In order to extract the exponential falloff of $E(n)$ which defines the correlation length, the pole structure of the integrand in the last term of (4.12) has to be analyzed. As can be seen from (A.2), (A.3) the $D_{j}(q), j=1,2$ involve cosine terms. Thus for the exact computation of the poles one has to solve transcendental equations, which cannot be done in closed form. As in the case of the vectorlike Schwinger model (compare 13, 14) in the hybrid approach one is reduced to a perturbative analysis for small $\mathrm{m}^{2}$. In lowest order one has to compute the zeros of $\sigma(q)$ in the first Brillouin zone $\left(B Z_{1}\right)$. From its definition (4.14) it is clear that $\sigma(p)$ becomes zero either if $D_{1}(q)$ vanishes, or if $D_{2}(q)$ approaches $\infty$. Inspecting (A.2) one immediately finds that $D_{1}(q)$ has no zeros in $B Z_{1}$. From (A.3) it can be seen that $D_{2}(q)$ becomes infinite only for $q \rightarrow 0$. However for $q \rightarrow 0, D_{1}(q) \rightarrow \infty$ as well, and one has to count powers. Using the infrared behaviour of $D_{j}(q), j=1,2$ (Lemma 2 in the Appendix), one finds

$$
\sigma(q)=\frac{q^{2}}{q_{1}^{2} q_{2}^{2}}\left(1+O\left(q^{2}\right)\right)\left[\frac{1}{q_{1}^{2} q_{2}^{2}}\left(1+O\left(q^{2}\right)\right)\right]^{-1}=q^{2}\left(1+O\left(q^{2}\right)\right) .
$$

Thus $\sigma(q)$ has only one zero in $B Z_{1}$ (at $q=0$ ), and Equation (4.15) displays the behaviour of $\sigma(q)$ around it. For small $m$, the integrand in (4.12) has a pole at

$$
q^{2}=-m^{2}\left(1+O\left(m^{2}\right)\right)
$$

This behaviour suggests that for small $m$ the model has a correlation length

$$
\xi=m^{-1}=\frac{1}{g} \frac{\sqrt{a-1}}{a} .
$$

$\xi$ becomes infinite for $g \rightarrow 0$ and arbitrary values of $a \geq 2$ (note that we already excluded values $a<2$ ). Of course the perturbative analysis given above does not prove that (4.17) holds. However there is a more elegant way, since for this simple model the continuum limit can be controlled analytically. For the moment we assume that $\xi$ is given by (4.17) and show that this assumption gives the correct continuum limit. 
We define our length scale $L_{0}$ to be proportional to the correlation length, i.e. $L_{0}:=\lambda \xi$. A physical distance $|x|$ is measured in units of $L_{0}$ giving rise to $x:=n / L_{0}$. The continuum gauge coupling $g_{c}\left(g_{c}=e_{c} / \sqrt{2 \pi}\right)$, which has the dimension of a mass is defined as $g_{c} a_{c} / \sqrt{a_{c}-1}:=$ $L_{0} g a / \sqrt{a-1}$. We also included a Jackiw-Rajaraman parameter $a_{c}$ for the continuum to match the continuum result completely. Putting things together, we obtain for the ratio $n / \xi$

$$
\frac{n}{\xi}=n g \frac{a}{\sqrt{a-1}}=n m=\text { const }=x g \frac{a_{c}}{\sqrt{a_{c}-1}}=: x m_{c} .
$$

We now perform the continuum limit by sending $\xi \rightarrow \infty,(\Leftrightarrow g \rightarrow 0)$ keeping $n / \xi$ fixed, i.e. we take the joint limit $g \rightarrow 0, n \rightarrow \infty$ in the sense of (4.18). We will show that this limit reproduces the continuum result for the two point function of the field strength. The continuum result reads (it can be computed easily using (2.10))

$$
\left\langle F_{12}(x) F_{12}(0)\right\rangle_{\text {cont }}=\delta^{(2)}(x)-\frac{m_{c}^{2}}{2 \pi} \mathrm{K}_{0}\left(m_{c}|x|\right) .
$$

We define

$$
I(n, g)=\int_{-\pi}^{\pi} \frac{d^{2} q}{(2 \pi)^{2}} \frac{e^{i q n}}{\sigma(q)+m^{2}} \quad, \quad J(n, g)=\int_{-\pi}^{\pi} \frac{d^{2} q}{(2 \pi)^{2}} \frac{e^{i q n}}{q^{2}+m^{2}} .
$$

$I(n, g)$ is just the integral in the last term of the expression for the two point function of $F_{12}$ on the lattice. The main step in controlling the continuum limit is to show that $d(n, g)$ defined as

$$
d(n, g):=I(n, g)-J(n, g)=\int_{-\pi}^{\pi} \frac{d^{2} q e^{i q n}}{(2 \pi)^{2}} \frac{q^{2}-\sigma(q)}{\left(\sigma(q)+m^{2}\right)\left(q^{2}+m^{2}\right)},
$$

vanishes in the joint limit $g \rightarrow 0, n \rightarrow \infty$ taken in the sense of (4.18). Since $\sigma(q)$ becomes zero only at $q=0, d(n, 0)$ exists (use (4.15) to see that the infrared singularity cancels), and

$$
\lim _{n \rightarrow \infty} d(n, 0)=0
$$

due to the Riemann-Lebesgue lemma. Using the triangle inequality

$$
|d(n, g)| \leq|d(n, g)-d(n, 0)|+|d(n, 0)|
$$

the problem is reduced to showing that $|d(n, g)-d(n, 0)| \rightarrow 0$ when performing the joint limit $g \rightarrow 0, n \rightarrow \infty$.

$$
|d(n, g)-d(n, 0)| \leq m^{2} \int_{-\pi}^{\pi} \frac{d^{2} q}{(2 \pi)^{2}}\left|\frac{1-q^{2} / \sigma(q)}{q^{2}}\right|\left|\frac{q^{2}+\sigma(q)+m^{2}}{\left(\sigma(q)+m^{2}\right)\left(q^{2}+m^{2}\right)}\right| .
$$

The first factor in the integrand could become unbounded only for $q \rightarrow 0$ or $\sigma(q) \rightarrow 0$. For the first case $(q \rightarrow 0) \sigma(q)$ behaves as $q^{2}(1+O(q)$ ) (see (4.15)), and thus the factor is bounded for $q \rightarrow 0$. This already covers the second case, since $\sigma(q)$ has its only zero at $q=0$, as discussed above. Thus there exists a bound $A$ for the first factor. We obtain

$$
|d(n, g)-d(n, 0)| \leq m^{2} A \int_{-\pi}^{\pi} \frac{d^{2} q}{(2 \pi)^{2}}\left|\frac{q^{2}+\sigma(q)+m^{2}}{\left(\sigma(q)+m^{2}\right)\left(q^{2}+m^{2}\right)}\right|
$$




$$
=m^{2} A \int_{-\pi}^{\pi} \frac{d^{2} q}{(2 \pi)^{2}} \frac{1}{q^{2}+m^{2}}+m^{2} A \int_{-\pi}^{\pi} \frac{d^{2} q}{(2 \pi)^{2}} \frac{1}{q^{2}+m^{2}}\left|\frac{q^{2}}{\sigma(q)+m^{2}}\right| .
$$

The second factor in the integrand of the last integral in (4.25) also can be bounded using the properties of $\sigma(q)$.

$$
\left|\frac{q^{2}}{\sigma(q)+m^{2}}\right|=\left|\frac{q^{2} / \sigma(q)}{1+m^{2} / \sigma(q)}\right|=\left|\frac{q^{2}}{\sigma(q)}\right| \frac{1}{\sqrt{1+2 \operatorname{Re} m^{2} / \sigma(q)+m^{4} /|\sigma(q)|^{2}}} .
$$

The first factor $\left|q^{2} / \sigma(q)\right|$ can be seen to be bounded, since $\sigma(q)$ becomes zero only at $q=0$ and behaves as $q^{2}(1+O(q))$ there (see (4.15)). Thus there exists some bound $B$ with $\left|q^{2} / \sigma(q)\right| \leq B$ for $q \in B Z_{1}$. In the Appendix it is shown that $D_{2}(q)$ has a positive real part (see Lemma 1). Using the fact that $D_{1}(q)$ is real and positive (see (A.2)) and the definition (4.14) of $\sigma(q)$, one concludes that $m^{2} / \sigma(q)$ has a positive real part. Thus the argument of the square root in the last term in (4.26) is greater 1 . The last factor in (4.26) is bounded by 1 , and the whole term by $B$. Putting things together, one obtains

$$
\begin{gathered}
|d(n, g)-d(n, 0)| \leq m^{2} A(1+B) \int_{-\pi}^{\pi} \frac{d^{2} q}{(2 \pi)^{2}} \frac{1}{q^{2}+m^{2}} \\
\leq \frac{m^{2} A(1+B)}{(2 \pi)^{2}} \int_{0}^{2 \pi} d \varphi \int_{0}^{2 \pi} d r \frac{r}{r^{2}+m^{2}}=\frac{m^{2} A(1+B)}{4 \pi}\left[\ln \left(4 \pi^{2}+m^{2}\right)-\ln \left(m^{2}\right)\right] \stackrel{g \rightarrow 0}{\longrightarrow} 0 .
\end{gathered}
$$

We used the fact that the integrand is positive, and extended the area of integration to a circle of radius $2 \pi$ around the origin. Inserting (4.22) and (4.27) into (4.23) establishes that $|d(n, g)| \rightarrow 0$ or equivalently $I(n, g) \rightarrow J(n, g)$ when performing the joint limit $g \rightarrow 0, n \rightarrow \infty$ in the sense of (4.18). Finally, using the transformation $p:=q / m$ of the integration variable, $J(n, g)$ can be rewritten to (use (4.18))

$$
J(n, g)=\int_{-\pi / m}^{\pi / m} \frac{d^{2} p}{(2 \pi)^{2}} \frac{e^{i p x m_{c}}}{p^{2}+1}=\frac{1}{2 \pi} \mathrm{K}_{0}\left(m_{c}|x|\right)+O(g) .
$$

We conclude

$$
I(n, g) \longrightarrow \frac{1}{2 \pi} \mathrm{K}_{0}\left(m_{c}|x|\right),
$$

when taking the joint limit $g \rightarrow 0, n \rightarrow \infty$. The last step is to remove the extra factor $m^{2}$ which shows up in (4.12) in front of $I(n, g)$ with a wave function renormalization constant

$$
Z_{12}(g):=\frac{m_{c}^{2}}{m^{2}}
$$

This also reproduces the contact term of the continuum result (4.19), since $\delta_{n_{1}, 0} \delta_{n_{2}, 0} m_{c}^{2} / m^{2} \rightarrow$ $\delta^{(2)}(x)$ for $g \rightarrow 0, n \rightarrow \infty$ with $n m=x m_{c}$ held fixed. Thus we have established

$$
\lim _{g \rightarrow 0, n \rightarrow \infty} Z_{12}(g)\left\langle F_{12}(n) F_{12}(0)\right\rangle=\left\langle F_{12}(x) F_{12}(0)\right\rangle_{c o n t} .
$$

It has to be remarked that the result (4.31) for the continuum limit is an exact result, and contains no more perturbation expansion. It shows, that the model has critical points where the continuum limit can be performed at $g=0, a \geq 2$. 


\section{$5 \quad$ Remarks on fermionic observables}

Since the fermions are treated in the continuum, the continuum fermion propagator in an external interpolated field has to be computed. The continuum propagator $G(x, y ; A)$ has to obey the Greens function equation

$$
\left[\frac{\partial}{\partial x_{1}}-i \frac{\partial}{\partial x_{2}}-i e\left(A_{1}(x)-i A_{2}(x)\right)\right] G(x, y ; A)=\delta^{(2)}(x-y) .
$$

A solution can be found easily by using Schwinger's original ansatz [25]

$$
G(x, y ; A):=G_{0}(x-y) \exp (i e[\Phi(x)-\Phi(y)])
$$

where $G_{0}$ denotes the propagator for free, left handed fermions which obeys

$$
\left[\partial_{1}-i \partial_{2}\right] G_{0}(x)=\delta^{(2)}(x-y)
$$

and $\Phi(x)$ is a solution of

$$
\left[\partial_{1}-i \partial_{2}\right] \Phi_{0}(x)=A_{1}(x)-i A_{2}(x) .
$$

Using $\left[\partial_{1}-i \partial_{2}\right]\left[\partial_{1}+i \partial_{2}\right]=\triangle$ and the Greens function $C(x)$ of $-\triangle$ given by $C(x)=-\ln \left(\mu^{2} x^{2}\right) / 4 \pi$ ( $\mu$ parametrizes the freedom in the infrared regularization) one obtains

$$
G_{0}(x)=-\left[\partial_{1}+i \partial_{2}\right] C(x)=\frac{1}{2 \pi}\left[x_{1}-i x_{2}\right]^{-1},
$$

and

$$
\begin{aligned}
\Phi(x) & =-\int_{-\infty}^{\infty} d^{2} z C(x-z)\left[\frac{\partial}{\partial z_{1}}+i \frac{\partial}{\partial z_{2}}\right]\left[A_{1}(z)-i A_{2}(z)\right] \\
& =-\int_{-\infty}^{\infty} \frac{d^{2} p}{(2 \pi)^{2}} \frac{e^{i p x}}{p^{2}}\left[i p_{1}-p_{2}\right]\left[\widetilde{A}_{1}(p)-i \widetilde{A}_{2}(p)\right]
\end{aligned}
$$

Inserting the interpolated gauge field $\widetilde{A}_{\mu}^{\text {int }}(2.6)$ and restricting the arguments $x, y$ to lattice points $n, m$, one ends up with

$$
G\left(n, m ; A^{i n t}\right)=\frac{1}{2 \pi}\left[\left(n_{1}-m_{1}\right)-i\left(n_{2}-m_{2}\right)\right]^{-1} \exp \left(i e\left[\Phi^{i n t}(n)-\Phi^{i n t}(m)\right]\right)
$$

where

$$
\begin{aligned}
\Phi^{i n t}(n):= & \int_{-\pi}^{\pi} \frac{d^{2} q e^{i q n}}{(2 \pi)^{2}}\left[\widehat{A}_{1}(q) \sum_{k \in \mathbb{Z}} \frac{2-2 \cos \left(q_{2}\right)}{r_{2}^{2}} \frac{e^{-i q_{1}}-1}{r_{1}} \frac{r_{1}+i r_{2}}{r^{2}}\right. \\
& \left.-i \widehat{A}_{2}(q) \sum_{k \in \mathbb{Z}} \frac{2-2 \cos \left(q_{1}\right)}{r_{1}^{2}} \frac{e^{-i q_{2}}-1}{r_{2}} \frac{r_{1}+i r_{2}}{r^{2}}\right] .
\end{aligned}
$$

Again the integral over $\mathbb{R}^{2}$ had to be rewritten as an integral over the first Brillouin zone only, since the lattice fields and not the continuum fields are integrated over in the path integral.

The basic, gauge invariant fermionic observable is

$$
\chi(n) U_{\mathcal{C}}(n, m) \overline{\chi(m)},
$$


where $U_{\mathcal{C}}$ denotes a gauge transporter along some contour $\mathcal{C}$. It has to be remarked that one cannot build $U_{\mathcal{C}}$ out of the lattice gauge transporters (2.4). The reason is that such a simple construct $\left(U_{\mathcal{C}}^{\text {naive }}\right)$ transforms under a lattice gauge transformation

$$
A_{\mu}(n) \longrightarrow A_{\mu}(n)+\Lambda\left(n+\hat{e}_{\mu}\right)-\Lambda(n),
$$

as

$$
U_{\mathcal{C}}^{\text {naive }}(n, m) \longrightarrow e^{-i e \Lambda(n)} U_{\mathcal{C}}^{\text {naive }} e^{i e \Lambda(m)},
$$

while the propagator $G\left(n, m ; A^{i n t}\right)$ transforms as

$$
G\left(n, m ; A^{i n t}\right) \longrightarrow e^{i e \Delta(n)} G\left(n, m ; A^{i n t}\right) e^{-i e \Delta(m)},
$$

with

$$
\Delta(n):=\int_{-\pi}^{\pi} \frac{d^{2} q e^{i q n}}{(2 \pi)^{2}} \frac{2-2 \cos \left(q_{1}\right)}{q_{1}^{2}} \frac{2-2 \cos \left(q_{2}\right)}{q_{2}^{2}} \widehat{\Lambda}(q) .
$$

Thus the naive lattice transporter does not make (5.9) gauge invariant under lattice gauge transformations. Instead one has to use the continuum gauge transporter along some contour on the lattice and evaluate it for the interpolated fields $A_{\mu}^{\text {int }}$

$$
U_{\mathcal{C}}(n, m)=\exp \left(i e \int_{\mathcal{C}} A_{\mu}^{i n t}(z) d z_{\mu}\right):=\exp \left(i e\left(A_{\mu}^{i n t}, j_{\mu}^{\mathcal{C}}\right)\right)
$$

For e.g. a rectangular contour $\mathcal{R}$ with length $2 s$ and height $s(s \in \mathbb{N})$ one obtains

$$
\begin{aligned}
& \widehat{j_{1}^{\mathcal{R}}}(q):=-i 2 \sum_{k \in \mathbb{Z}} \frac{2-2 \cos \left(q_{2}\right)}{r_{2}^{2}} \frac{e^{i q_{1}}-1}{r_{1}} \frac{\sin \left(q_{1} s\right)}{r_{1}} e^{-i q_{2} s}, \\
& \widehat{j_{2}^{\mathcal{R}}}(q):=-i 2 \sum_{k \in \mathbb{Z}} \frac{2-2 \cos \left(q_{1}\right)}{r_{1}^{2}} \frac{e^{i q_{2}}-1}{r_{2}} \sin \left(q_{1} s\right) \frac{1-e^{-i q_{2} s}}{r_{2}} .
\end{aligned}
$$

Thus for computing expectation values of products of (5.9), only Gaussian functional integrals have to be solved. However the results become rather involved, since in the covariance (4.10) as well as in the exponentials of propagator (5.7) and gauge transporter (5.14) sums over all Brillouin zones occur.

We conclude this section with computing at least one fermionic expectation value, namely one where the integration over the gauge fields becomes trivial. We consider the two point function of the chiral density

$$
\langle\chi(n) \bar{\chi}(n) \chi(0) \bar{\chi}(0)\rangle^{c},
$$

where the superscript $c$ stands for connected. One finds

$$
\langle\chi(n) \bar{\chi}(n) \chi(0) \bar{\chi}(0)\rangle^{c}=-\int \frac{D[A]}{Z} G\left(0, n ; A^{i n t}\right) G\left(n, 0 ; A^{i n t}\right) .
$$

$D[A] / Z$ denotes a normalized functional integral over the lattice gauge fields, which can e.g. be realized as a Gaussian integral over $\varphi_{\mu}$ with covariance (4.10), as was done in the last section. However, by inspecting (5.7), one finds that the dependence on the gauge fields cancels for the 
quoted product of propagators and integration over the gauge fields simply gives a factor 1 . We end up with

$$
\langle\chi(n) \bar{\chi}(n) \chi(0) \bar{\chi}(0)\rangle^{c}=-\frac{1}{(2 \pi)^{2}}\left[n_{1}-i n_{2}\right]^{-2} .
$$

As can be seen from this result, the two point function of the chiral density clusters as in the continuum model. No condensate is being formed. This behaviour is different from the vectorlike Schwinger model where the expectation value of the chiral density is known to be nonvanishing, in the continuum as well as in hybrid approach [13.

From (4.18) and (5.18) it can be seen that again just a wave function renormalization is necessary in the continuum limit. Define

$$
Z_{\chi \bar{\chi}}(g):=\frac{m_{c}^{2}}{m^{2}}
$$

One ends up with

$\lim _{g \rightarrow 0, n \rightarrow \infty} Z_{\chi \bar{\chi}}(g)\langle\chi(n) \bar{\chi}(n) \chi(0) \bar{\chi}(0)\rangle^{c}=\langle\chi(x) \bar{\chi}(x) \chi(0) \bar{\chi}(0)\rangle_{c o n t}^{c}=-\frac{1}{(2 \pi)^{2}}\left[x_{1}-i x_{2}\right]^{-2}$,

where the limit was taken in the sense of (4.18)

\section{Concluding remarks}

It has been demonstrated that the hybrid approach works rather well for the chiral Schwinger model. The effective lattice gauge theory was constructed using the Jackiw-Rajaraman determinant for the chiral fermions in a background field. The resulting effective lattice gauge theory was proven to be Osterwalder-Schrader positive, for both the non-compact and the Wilson formulation. For the non-compact formulation we established the existence of critical points for zero gauge coupling and Jackiw-Rajaraman parameter $a \geq 2$. The continuum limit was performed explicitly for the two point functions of field strength and chiral densities.

As a next step it might be interesting to explore the hybrid approach for a model with nonAbelian gauge group. A possible candidate could be $\mathrm{QCD}_{2}$ where reasonable explicit definitions of regularized continuum fermion determinants in a background field exist (for an overview see e.g. [26]). Even more challenging is of course the extension to four dimensional theories. The problem there is that the determinant is either given very implicitly or only the first few terms of a loop expansion are known. An approach that is promising at least for vectorlike theories is the introduction of two independent lattice cutoffs, a coarse lattice for the gauge fields and a finer lattice for the fermions [12]. It should be possible to proof OS positivity for this setting by conventional methods [23].

\section{Ackknowledgement :}

The author thanks Erhard Seiler for his ongoing interest in this project and valuable discussions, and Peter Weisz for reading the manuscript. 


\section{Appendix}

In the Appendix we analyze the properties of the determinant of the kernel $\widehat{C}^{-1}(q)$ of the effective action (4.6). The determinant of the matrix $\widehat{C}^{-1}(q)$ can be written as

$$
D(q):=\operatorname{det} \widehat{C}^{-1}(q):=g^{2}(a+1) D_{1}(q)+g^{4} a^{2} D_{2}(q) .
$$

The terms $D_{j}(q), j=1,2$ are given by

$$
D_{1}(q)=\sum_{k \in \mathbb{Z}^{2}} \frac{2-2 \cos \left(r_{1}\right)}{r_{1}^{2}} \frac{2-2 \cos \left(r_{2}\right)}{r_{2}^{2}} \frac{r^{2}}{r_{1}^{2} r_{2}^{2}},
$$

and

$$
\begin{gathered}
D_{2}(q):=\frac{1}{a^{2}} \sum_{k, l \in \mathbb{Z}} \frac{\left(2-2 \cos \left(q_{1}\right)\right)^{2}}{r_{1}^{2} s_{1}^{2}} \frac{\left(2-2 \cos \left(q_{2}\right)\right)^{2}}{r_{2}^{2} s_{2}^{2}} \frac{1}{r_{1}^{2} r_{2}^{2} r^{2}} \frac{1}{s_{1}^{2} s_{2}^{2} s^{2}} \\
\times\left\{\left[(a+1) r_{2}^{2} r^{2}-2 r_{2}^{4}\right]\left[(a+1) s_{1}^{2} s^{2}-2 s_{1}^{4}\right]+4 r_{1} s_{1}^{3} r_{2}^{3} s_{2}-4 r_{1}^{2} s_{1}^{2} r_{2}^{2} s_{2}^{2}+\left(r_{1}^{3} r_{2}-r_{1} r_{2}^{3}\right)\left(s_{1}^{3} s_{2}-s_{1} s_{2}^{3}\right)\right. \\
\left.+i 2\left[r_{1} r_{2}^{3}\left((a+1) s_{1}^{2} s^{2}-2 s_{1}^{4}\right)-s_{1}^{3} s_{2}\left((a+1) r_{2}^{2} r^{2}-2 r_{2}^{4}\right)+r_{1}^{2} r_{2}^{2}\left(s_{1}^{3} s_{2}-s_{1} s_{2}^{3}\right)+s_{1}^{2} s_{2}^{2}\left(r_{1}^{3} r_{2}-r_{1} r_{2}^{3}\right)\right]\right\} .
\end{gathered}
$$

We introduced the abbreviations

$$
r_{\mu}:=q_{\mu}+2 \pi k_{\mu} \quad, \quad s_{\mu}:=q_{\mu}+2 \pi l_{\mu} .
$$

Lemma 1: The real part of $D(q)$ is strictly positive for $a \geq 2$ and $g>0$.

From (A.2) it is obvious, that $D_{1}(q)$ is real and strictly positive. Thus there is left to show, that $D_{2}(q)$ has a positive real part. The real part of $D_{2}(q)$ is the sum over

$$
S:=\left[(a+1) r_{2}^{2} r^{2}-2 r_{2}^{4}\right]\left[(a+1) s_{1}^{2} s^{2}-2 s_{1}^{4}\right]+4 r_{1} s_{1}^{3} r_{2}^{3} s_{2}-4 r_{1}^{2} s_{1}^{2} r_{2}^{2} s_{2}^{2}+\left(r_{1}^{3} r_{2}-r_{1} r_{2}^{3}\right)\left(s_{1}^{3} s_{2}-s_{1} s_{2}^{3}\right),
$$

with the positive weight

$$
\frac{\left(2-2 \cos \left(q_{1}\right)\right)^{2}}{r_{1}^{2} s_{1}^{2}} \frac{\left(2-2 \cos \left(q_{2}\right)\right)^{2}}{r_{2}^{2} s_{2}^{2}} \frac{1}{r_{1}^{2} r_{2}^{2} r^{2}} \frac{1}{s_{1}^{2} s_{2}^{2} s^{2}} .
$$

Note that the weight is symmetric with respect to $r$ and $s$. This allows to interchange the role of $r$ and $s$ in individual terms of $S$ under the sum. Using this trick one can rewrite $S$ to

$$
S=\left[(a-1)^{2}-1\right] r^{2} s^{2} r_{1}^{2} s_{2}^{2}+2(a-1) r_{1}^{2} r_{2}^{2}\left(s^{2}\right)^{2}+\frac{1}{2} r^{2} s^{2}\left(r_{1} s_{2}+r_{2} s_{1}\right)^{2} .
$$

Equation (A.7) makes it obvious, that each term in $D_{2}(q)$ has a positive real part for $a \geq 2$, and thus Lemma 1 is proven.

Lemma 1 implies that the determinant $D(q)$ does not vanish for $a \geq 2$, and that the propagator $C(q)$ for the fields $\varphi_{\mu}$ exists for that range of $a$. It also implies, that the propagator for the original gauge fields exists. From (4.4) it can be seen, that the determinant of the kernel for the action written in terms of $A_{\mu}$ differs from $D(q)$ by an overall factor $\left[2-2 \cos \left(q_{1}\right)\right]\left[2-2 \cos \left(q_{2}\right)\right]$. 
This factor is positive and vanishes in the first Brillouin zone only at $q=0$. The corresponding zero is cancelled in $D_{1}(q)$ and $D_{2}(q)$ in at least the $k=0$ terms, which is sufficient to obtain a determinant with strictly positive real part. Thus the kernel in the action written in terms of the gauge fields can be inverted for $a \geq 2$ as well, giving the propagator for the gauge field $A_{\mu}$.

Lemma 2: The terms $D_{j}(q), j=1,2$ have the following infrared behaviour:

$$
D_{1}(q)=\frac{q^{2}}{q_{1}^{2} q_{2}^{2}}\left[1+O\left(q^{2}\right)\right] \quad, \quad D_{2}(q)=\frac{1}{q_{1}^{2} q_{2}^{2}}\left[1+O\left(q^{2}\right)\right] .
$$

From the definition (A.2) it is clear that the most singular term in the sum for $D_{1}(q)$ is the term with $k=0$. It behaves as $q^{2} /\left(q_{1}^{2} q_{2}^{2}\right)$. All other terms have at least an extra factor $q_{\mu}^{2}$ which already establishes the first part of (A.8). The infrared behaviour of $D_{2}(q)$ is a little bit more involved to analyze. $D_{2}(q)$ can be rewritten as the sum of

$$
\sum_{k, l \in \mathbb{Z}} \frac{\left(2-2 \cos \left(q_{1}\right)\right)^{2}}{r_{1}^{2} s_{1}^{2}} \frac{\left(2-2 \cos \left(q_{2}\right)\right)^{2}}{r_{2}^{2} s_{2}^{2}} \frac{1}{r_{1}^{2} s_{2}^{2}},
$$

and

$$
\begin{gathered}
\frac{1}{a^{2}} \sum_{k, l \in \mathbb{Z}} \frac{\left(2-2 \cos \left(q_{1}\right)\right)^{2}}{r_{1}^{2} s_{1}^{2}} \frac{\left(2-2 \cos \left(q_{2}\right)\right)^{2}}{r_{2}^{2} s_{2}^{2}} \frac{1}{r_{1}^{2} r_{2}^{2} r^{2}} \frac{1}{s_{1}^{2} s_{2}^{2} s^{2}} \\
\times\left\{2 a r_{1}^{2} s_{2}^{2}\left(s_{1}^{2} r_{2}^{2}-r_{1}^{2} s_{2}^{2}\right)-r_{1}^{2} r_{2}^{2} s_{2}^{4}+r_{1} s_{1} r_{2}^{3} s_{2}^{3}-r_{1}^{4} s_{1}^{2} s_{2}^{2}+r_{1}^{3} s_{1}^{3} r_{2} s_{2}+r_{1}^{4} s_{2}^{4}-3 r_{1}^{2} s_{1}^{2} r_{2}^{2} s_{2}^{2}\right. \\
\left.+2 r_{1}^{3} s_{1} r_{2} s_{2}^{3}+i 2(a-1)\left[r_{1}^{4} s_{1} s_{2}^{3}-r_{1}^{3} s_{1}^{2} r_{2} s_{2}^{2}+r_{1}^{2} s_{1} r_{2}^{2} s_{2}^{3}-r_{1}^{3} r_{2} s_{2}^{4}\right]\right\} .
\end{gathered}
$$

The first part (A.9) is easily seen to have its most singular term at $k=l=0$ and it behaves as $1 /\left(q_{1}^{2} q_{2}^{2}\right)$. The second term (A.10) is more subtle. For $k=l=0$, the contribution adds up to zero, and the potentially most singular term is not there. The terms $k_{\mu} \neq 0 ; l_{\mu} \neq 0 ; k_{1}, k_{2} \neq 0 ; l_{1}, l_{2} \neq 0$ and $k_{\mu}, l_{\nu} \neq 0$ can be seen to be of $O\left(q^{2}\right)$ less singular than $1 /\left(q_{1}^{2} q_{2}^{2}\right)$. This establishes the second part of (A.8).

\section{References}

[1] K. G. Wilson, Phys. Rev. D 10 (1974) 2445.

[2] Y. Shamir, Review talk given at Lattice 95, Report No. hep-lat/9509023.

[3] H.B. Nielsen, M. Ninomya, Nucl. Phys. B 185 (1981) 20;

Nucl. Phys. B 193 (1981) 173.

[4] G. 't Hooft, Phys. Lett. 349 B (1995) 491.

[5] R. Flume, D. Wyler, Phys. Lett. 108 B (1982) 317.

[6] R. L. Stuller, "Hybrid Quantization", unpublished notes. 
[7] M. Göckeler, G. Schierholz, Nucl. Phys. (Proc. Suppl.) 29 B,C (1992) 114;

Nucl. Phys. (Proc. Suppl.) 30 B (1993) 609.

[8] M. Göckeler, A.S. Kronfeld, G. Schierholz, U.-J. Wiese, Nucl. Phys. B 404 (1993) 839.

[9] S.D.H. Hsu, Report No. hep-th/9503058 (1995).

[10] I. Montvay, Report No. hep-lat/9505015 (1995).

[11] A.S. Kronfeld, Report No. hep-lat/9504007 (1995).

[12] P. Hernandez, R. Sundrum, Nucl. Phys. B 455 (1995) 287;

Report No. hep-ph/9510328 (1995);

Report No. hep-lat/9602017 (1996).

[13] C. Gattringer, Report No. hep-lat/9511019, to appear in May 1. issue of Phys. Rev. D (1996).

[14] C. Gattringer, Report No. hep-lat/9603010, to appear in the proceedings of 'Hadron Structure 96', Feb. 11-16, 1996, Stara Lesna, Slovakia.

[15] K. Osterwalder, R. Schrader, Commun. Math. Phys. 31 (1973) 83;

Commun. Math. Phys. 42 (1975) 281.

[16] R. Jackiw, R. Rajaraman, Phys. Rev. Lett. 54 (1985) 1219.

[17] R. Rajaraman, Phys. Lett. 154 B (1985) 305.

[18] A.J. Niemi, G.W. Semenoff, Phys. Lett. 175 B (1986) 439.

[19] H.O. Girotti, H.J. Rothe, K.D. Rothe, Phys. Rev. D 33 (1986) 514;

Phys. Rev. D 34 (1986) 592.

[20] C.A. Linhares, H.J. Rothe, K.D. Rothe, Phys. Rev. D 37 (1988) 427.

[21] L. Alvarez-Gaumé, S. Della Pietra, V. Della Pietra, Phys. Lett. 166 B (1986) 177.

[22] R. Banerjee, Phys. Rev. Lett. 56 (1986) 1889.

[23] K. Osterwalder, E. Seiler, Ann. Phys. 110 (1978) 440.

[24] J. Fröhlich, R. Israel, E.H. Lieb, B. Simon, Commun. Math. Phys. 62 (1978) 1;

J. Stat. Phys. 22 (1980) 297.

[25] J. Schwinger, Phys. Rev. 128 (1962) 2425.

[26] E. Abdalla, M.C.B. Abdalla, K.D. Rothe, Nonperturbative Methods in 2 Dimensional Quantum Field Theory, World Scientific (1991), Singapore, New Jersey, London, Hong Kong. 